\title{
The valence band structure of Gadolinium studied with time-resolved photoemission
}

\author{
B. Frietsch, J. Bowlan, R. Carley, M. Teichmann, J. Wolter, and M. Weinelt
}

Fachbereich Physik, Freie Universität Berlin, Arnimallee 14, 14195 Berlin, Germany and Max-Born-Institut, Max-Born-Strasse 2A, 12489 Berlin, Germany

Author e-mail address: weinelt@physik.fu-berlin.de

\begin{abstract}
We have studied the response of the exchange split valence bands of ferromagnetic gadolinium to femtosecond laser excitation. We observe a drop of the exchange splitting with a time constant of 0.9 ps but different response times of minority and majority spin bands. Furthermore, even above the Curie temperature there is a finite exchange splitting, which also decreases with laser excitation.
\end{abstract}

\section{Introduction}

It is well established that optical excitation of thin ferromagnetic films leads to demagnetization within a few hundred femtoseconds [1] and even ultrafast optical magnetic switching of ferrimagnets has been demonstrated [2]. However, it remains controversial which microscopic processes are fast enough to provoke femtomagnetism: direct interaction with the laser field [3], scattering among electrons [4], phonons [5], and magnons [6], and/or spin-transport [7]. To establish microscopic models it is necessary to unravel the transient electronic structure [8] and clarify on which timescale the exchange splitting $\Delta \mathrm{E}_{\mathrm{ex}}$ and spin polarization collapses.

To approach these problems we perform time- and angle-resolved photoemission (TR-ARPES). We use laser-driven high-order harmonic generation as an extreme ultraviolet light source [9]. A Ti:Sapphire CPA laser system delivers $1.5 \mathrm{~mJ}$, $40 \mathrm{fs}$ IR pulses at $10 \mathrm{kHz}$ repetition rate, which are focused into a gas cell containing argon at 100 mbar. Individual harmonics are selected by a toroidal grating monochromator (TGM) with a single grating. The time resolution $\tau \sim \lambda / c N$ is primarily determined by the transmitted bandwidth ( $N$ is the number of illuminated lines). While the temporal spread is nearly independent of the TGM slit-size, the latter allows tuning of the energy resolution $\Delta E$. For $10 \mu \mathrm{m}$ slits we obtain $\tau=100 \mathrm{fs}, \Delta E=150 \mathrm{meV}$, and $2 \cdot 10^{4}$ photons/pulse at $h v=35.6 \mathrm{eV}$. 


\section{Gadolinium valence-band dynamics and exchange splitting}

We have studied ultrafast demagnetization of the local-moment ferromagnet gadolinium [10], prepared as epitaxial film of $10 \mathrm{~nm}$ thickness on a W(110) substrate. Photoelectrons are detected after a hemispherical energy analyzer. Tuning the TGM to $35.6 \mathrm{eV}$ allows us to map the valence bands of $\mathrm{Gd}$ at the $\Gamma$-point of the 4th Brillouin zone. Binding energy and exchange splitting of the $\Delta_{2}$-like $\Sigma$ bands are shown in Fig.1a. The initial binding energies of 1.39 and $2.25 \mathrm{eV}$ are in agreement with synchrotron measurements conducted at a comparable sample temperature of $100 \mathrm{~K}$ [11]. Upon laser excitation (hv = $1.6 \mathrm{eV}$, s-pol., $300 \mathrm{fs,} \mathrm{flu-}$ ence $1.2 \mathrm{~mJ} / \mathrm{cm}^{2}$ ) the exchange splitting of the valence bands drops with a time constant of $0.9 \pm 0.1 \mathrm{ps}$. This value coincides with the values observed in MOKE [12] and XMCD [13] measurements. The latter experiment probes the $4 f$ spin subsystem but was conducted at higher pump fluences $\left(3-5 \mathrm{~mJ} / \mathrm{cm}^{2}\right)$. Comparing ultrafast de- and thermal re-magnetization [10] we conclude that $\Delta \mathrm{E}_{\mathrm{ex}}$ maps the true magnetization. From Fig. 1a it is evident that the minority valence band reacts immediately after laser excitation while the response of its majority counterpart is delayed and is only half as fast. Delay zero was independently determined from the first occurrence of pump-induced hot electrons above the Fermi level $E_{F}$. We attribute the instantaneous response of the minority spin band to superdiffusive spin transport [7]. Photoemission probes the first few surface layers and is thus very sensitive to spin currents into the bulk. The delayed response of the majority band can be explained by electron-phonon scattering which increases with increasing lattice temperature [5]. Our data suggest that both spin transport and electronphonon scattering contribute to ultrafast demagnetization. In addition, the molecular field acting on the spins may delay the response of the majority spin band [14].

It has been a long standing question whether the exchange splitting vanishes at the Curie temperature $T_{C}=293 \mathrm{~K}$ [15]. For the Gd surface state scanning tunnel-
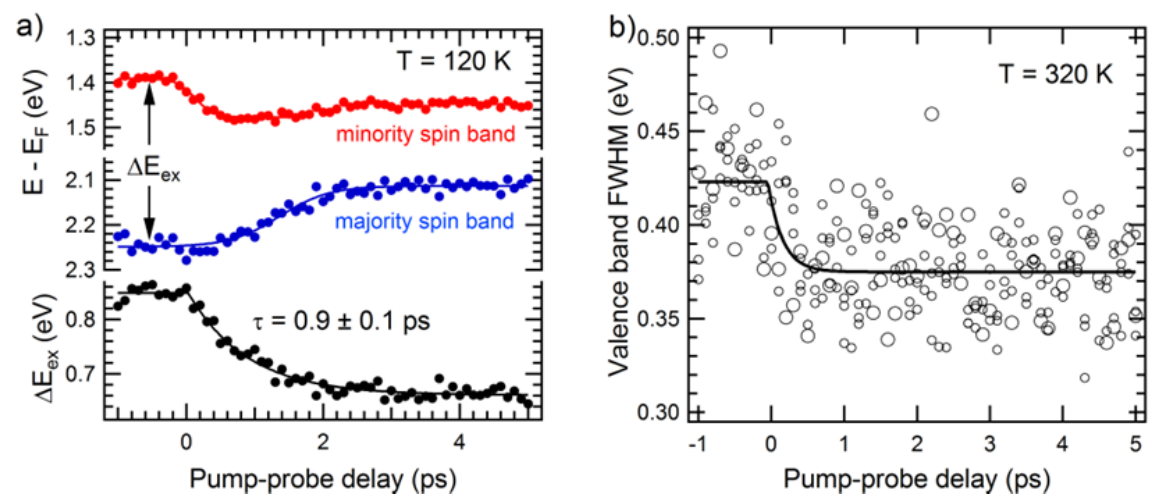

Fig. 1. a) Temporal evolution of the Gd minority and majority spin bands and exchange splitting upon optical excitation. b) Decrease of the valence band's full width at half maximum at $320 \mathrm{~K}$. 
ing spectroscopy revealed a sizeable exchange splitting at $T_{C}[16]$. However, photoemission measurements of the bulk valence bands remain ambiguous since the minority and majority spin bands coalesce into a single peak due to thermal broadening $[11,15]$. In Fig. 1b we show the linewidth of this peak as a function of pump-probe delay at $320 \mathrm{~K}$. Upon laser excitation we observe a small but significant decrease of the linewidth, which can only be explained by a further reduction of $\Delta \mathrm{E}_{\mathrm{ex}}$. Above $\mathrm{T}_{\mathrm{C}}$ there exists in fact a finite exchange splitting, which implies local magnetic order.

Acknowledgments We gratefully acknowledge funding by the Deutsche Forschungsgemeinschaft, the Leibniz graduate school DinL, and the Helmholtz Virtual Institute Dynamic Pathways in Multidimensional Landscapes. J.B. is indebted to the Humboldt Foundation for a scholarship.

[1] E. Beaurepaire, J.-C. Merle, A. Daunois, and J.-Y. Bigot, "Ultrafast Spin Dynamics in Ferromagnetic Nickel” Phys. Rev. Lett. 76, 4250 (1996).

[2] I. Radu et al., "Transient ferromagnetic-like state mediating ultrafast reversal of antiferromagnetically coupled spins” Nature 472, 205 (2011).

[3] J. Bigot, M. Vomir, and E. Beaurepaire, "Coherent ultrafast magnetism induced by femtosecond laser pulses” Nature Physics 5, 515 (2009).

[4] M. Krauß et al., "Ultrafast demagnetization of ferromagnetic transition metals: The role of the Coulomb interaction” Phys. Rev. B 80, 180407 (2009).

[5] B. Koopmans et al., "Explaining the paradoxical diversity of ultrafast laser-induced demagnetization” Nature Mater. 9, 259-265 (2010).

[6] A.B. Schmidt et al., „Ultrafast Magnon Generation in an Fe Film on $\mathrm{Cu}(100)$ “ Phys. Rev. Lett. 105, 197401 (2010).

[7] M. Battiato, K. Carva, and P. M. Oppeneer, "Superdiffusive Spin Transport as a Mechanism of Ultrafast Demagnetization” Phys. Rev. Lett. 105, 027203 (2010).

[8] A. J. Schellekens and B. Koopmans, "Comparing Ultrafast Demagnetization Rates Between Competing Models for Finite Temperature Magnetism” Phys. Rev. Lett. 110, 217204 (2013); K. Carva, M. Battiato, D. Legut, and P. M. Oppeneer, "Ab initio theory of electron-phonon mediated ultrafast spin relaxation of laser-excited hot electrons in transition-metal ferromagnets” Phys. Rev. B 87, 184425 (2013).

[9] B. Frietsch et al., "A high-order harmonic generation apparatus for time- and angle-resolved photoelectron spectroscopy” Rev. Sci. Instrum. 84 (2013), in press.

[10] R. Carley et al., "Femtosecond Laser Excitation Drives Ferromagnetic Gadolinium out of Magnetic Equilibrium” Phys. Rev. Lett. 109, 057401 (2012).

[11] C. Schüßler-Langeheine, "Magnetic properties of thin films of heavy lanthanide metals studied by magnetic x-ray diffraction and high-resolution photoemission,” Ph.D. thesis, Fachbereich Physik, Freie Universität Berlin, 1999.

[12] Magneto Optical Kerr Effect, M. Sultan et al., "Electron- and phonon-mediated ultrafast magnetization dynamics of Gd(0001)”, Phys. Rev. B 85, 184407 (2012).

[13] X-ray magnetic circular dichroism; $M$. Wietsruk et al., "Hot-electron-driven enhancement of spin-lattice coupling in Gd and $\mathrm{Tb} 4 \mathrm{f}$ ferromagnets observed by Femtosecond X-ray magnetic circular dichroism” Phys. Rev. Lett. 106, 127401 (2011).

[14] M.Avignon and K.-H. Bennemann, http://arxiv.org/pdf/1305.5698.pdf.

[15] K. Maiti, M. C. Malagoli, A. Dallmeyer, and C. Carbone, "Finite Temperature Magnetism in Gd: Evidence against a Stoner Behavior” Phys Rev. Lett. 88, 167205 (2002).

[16] M. Bode et al., "Temperature-Dependent Exchange Splitting of a Surface State on a LocalMoment Magnet: Tb(0001)”, Phys. Rev. Lett. 83, 3017 (1999). 